\title{
The Muon Accelerator Research and Development Program
}

\author{
Gail G. Hanson ${ }^{*}$ \\ Department of Physics and Astronomy, University of California \\ Riverside, California 92521, U.S.A. \\ E-mail: Gail.Hanson@ern.ch
}

\begin{abstract}
A muon accelerator facility that leads to a multi- $\mathrm{TeV}$ Muon Collider presents the unique opportunity to explore new physics within a number of distinct programs that can be brought online as the facility evolves. An introduction to the Muon Collider facility and its capabilities will be given. The Muon Accelerator Program, hosted by Fermilab, has recently been approved by the U.S. Department of Energy to carry out the Research and Development necessary to demonstrate the feasibility of the Muon Collider, including the technology development and system tests needed to inform the study, and to contribute to the International Design Study for a Neutrino Factory.
\end{abstract}

The 2011 Europhysics Conference on High Energy Physics-HEP2011 Grenoble, Rhône-Alpes

July 21-27 2011

Speaker

$\S \quad$ On behalf of the Muon Accelerator Program (MAP). 


\section{Why a Muon Collider?}

Colliding beams of positively- and negatively-charged muons in a storage ring have the same physics potential as an electron-positron collider.

The current results from the Large Hadron Collider (LHC) hint that higher center-of-mass energies than the $0.5 \mathrm{TeV}$ International Linear Collider (ILC) will be needed. So far, many limits on production of new physics particles exceed masses of $1 \mathrm{TeV}$. The center-of-mass energy of the proposed Compact Linear Collider (CLIC) is $3 \mathrm{TeV}$. A comparison of design parameters for 1.5 and $3 \mathrm{TeV}$ Muon Colliders and the $3 \mathrm{TeV}$ CLIC is given in table 1 . The wall power for the Muon Collider is estimated to be one-third that of the $3 \mathrm{TeV}$ CLIC and two-thirds that of the $0.5 \mathrm{TeV}$ ILC.

Muons have an advantage over electrons since synchrotron radiation is proportional to $(E / m)^{4}$, allowing a multi-TeV Muon Collider to be circular and compact. The large muon mass greatly reduces beamstrahlung, resulting in a much small collision energy spread than at a comparable energy $e^{+} e^{-}$collider. A multi-TeV Muon Collider can easily fit on the Fermilab site.

\begin{tabular}{|l|l|l|l|l|}
\hline \multicolumn{1}{|c|}{ Parameter } & \multicolumn{1}{c|}{ Unit } & $\boldsymbol{\mu}^{+} \boldsymbol{\mu}^{-}$ & $\boldsymbol{\mu}^{+} \boldsymbol{\mu}^{-}$ & $\boldsymbol{e}^{+} \boldsymbol{e}^{-}$CLIC \\
\hline Center-of-mass energy & $\mathrm{TeV}$ & 1.5 & 3 & 3 \\
\hline Luminosity & $10^{34} \mathrm{~cm}^{-2} \mathrm{~s}^{-1}$ & 1 & 4 & 2 \\
\hline Bending field (ring) & $\mathrm{T}$ & 6 & 8.4 & - \\
\hline $\begin{array}{l}\text { Accelerator } \\
\text { circumference/length }\end{array}$ & $\mathrm{km}$ & 6 & 12 & 48 \\
\hline rms bunch height & $\mu \mathrm{m}$ & 6 & 4 & 0.001 \\
\hline Proton beam power & $\mathrm{MW}$ & 4 & 3.2 & - \\
\hline Lepton power total & $\mathrm{MW}$ & 7 & 11 & 28 \\
\hline Wall power & $\mathrm{MW}$ & $\approx 147$ & $\approx 159$ & 560 \\
\hline
\end{tabular}

Table 1: Comparison of Muon Collider parameters with 3.0 TeV CLIC [1].

\section{The Muon Accelerator Program (MAP)}

The Muon Accelerator Program (MAP) [2], hosted by Fermilab, was formed to coordinate the R\&D that had been carried out by the Neutrino Factory and Muon Collider Collaboration (NFMCC) and the Muon Collider Task Force (MCTF) over 10 years. A review of the program was held at Fermilab in August 2010, and MAP was approved by the U.S. Department of Energy in March 2011. The search for a Director is underway.

The goals of MAP are to (1) complete a Design Feasibility Study (DFS) Report for a multi-TeV Muon Collider, including cost range; (2) contribute to the International Neutrino Factory Design Study (IDS-NF) [3] and produce a Reference Design Report by 2013; (3) carry out supporting technology R\&D needed to inform the Muon Collider DFS and enable downselection; and (4) participate in system tests of 4D and 6D cooling - the Muon Ionization 
Cooling Experiment (MICE) [4] and a 6D "bench test" (no beam). The MAP program should be completed over a time period of 6-7 years.

A schematic of the proposed Muon Collider layout is shown in figure 1. The first part of the layout is based on the IDS-NF scheme, re-optimized for the collider.

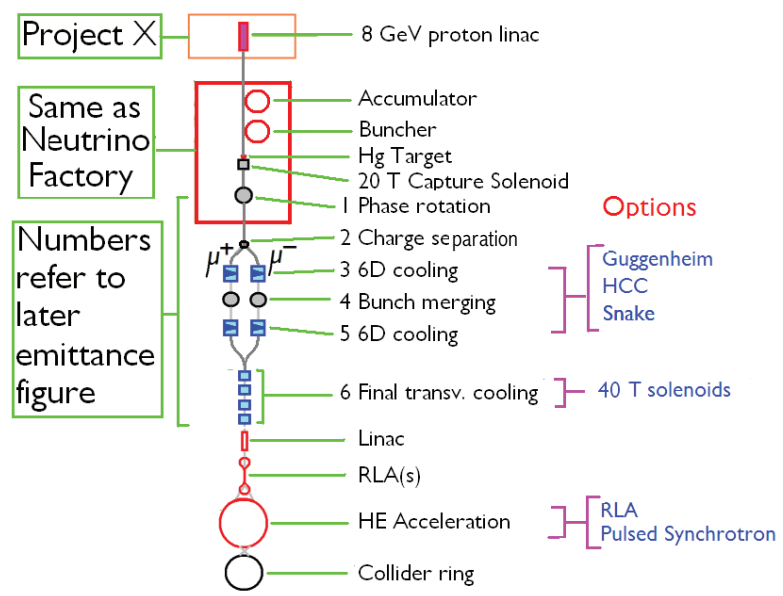

Figure 1: Schematic of a Muon Collider.

\subsection{Proton Source}

The high-intensity proton source is an upgrade of Project $\mathrm{X}$, producing an $8 \mathrm{GeV}$ beam of about $2 \times 10^{14}$ protons, in short bunches at a frequency of $15 \mathrm{~Hz}$, with a total power of $4 \mathrm{MW}$.

\subsection{Target and Capture}

The target is a liquid mercury jet inside a superconducting solenoid. A liquid metal jet can withstand the heating from the $4 \mathrm{MW}$ proton beam. The concept was successfully demonstrated in the MERIT experiment [5] at the CERN PS. Pions produced in the target are captured in a 20-T axial magnetic field generated by an inner copper coil and an outer superconducting solenoid. Radiation shielding is provided by water-cooled tungsten carbide beads.

\subsection{Phase Rotation and Buncher}

The pions produced in the target, and the muons into which they decay, have a very large energy spread but are produced over a short time interval, determined by the bunch length of the protons plus decay kinematics. The beam is allowed to drift, after which a system of RF cavities is used to bunch the distribution. Further RF cavities then phase rotate the bunches, decelerating the faster bunches and accelerating the slower ones. The beam is then separated into two beams of positive and negative charges.

\subsection{Cooling}

Ionization cooling is used to reduce the six-dimensional phase space of the muon beam. The cooling scheme is illustrated in figure 2. Three options are being considered for 6D cooling: the helical RFOFO "Guggenheim," the helical FOFO snake, and the Helical Cooling Channel (HCC) [6]. Simulations of the Guggenheim method are shown in figure 2. 


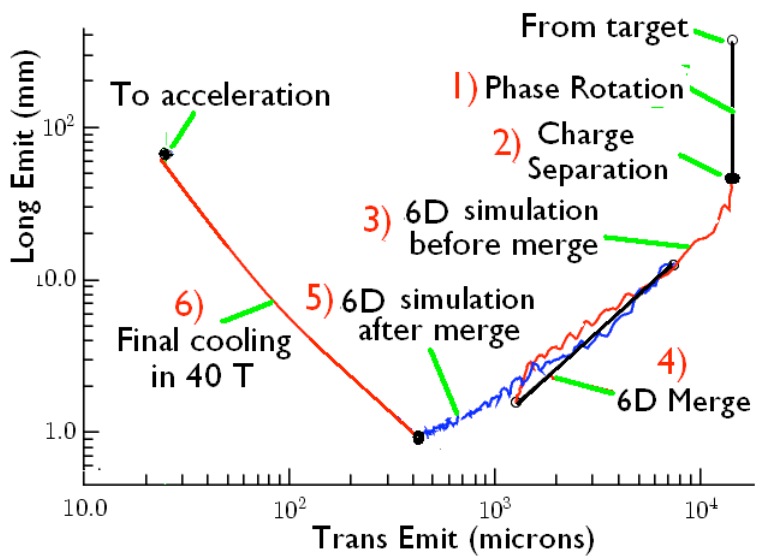

Figure 2: Cooling scheme from production to the start of acceleration.

The multiply-bunched beams of muons are then merged into a single channel, with single positive and negative bunches separated in time. After re-cooling, the resulting beam has a transverse emittance that is about 10 times larger than required but a longitudinal emittance that is almost 100 times smaller. The final cooling reduces the transverse emittance while increasing the longitudinal emittance using a system of high-field small-bore solenoids inside of which the muons pass through a liquid hydrogen absorber. A final transverse emittance of less than $25 \mu \mathrm{m}$ is achieved in simulation.

\subsection{Acceleration}

Low energy acceleration is achieved using a re-optimization of the IDS-NF scheme of a linac and two RLAs. Acceleration to high energy employs two pulsed rapid cycling synchrotrons; an alternative option would be one or more additional RLAs.

\subsection{Collider Ring}

The design of the collider ring takes into account two low- $\beta$ insertions for two detectors. As compared with existing colliders, the collider ring has much larger momentum acceptance with much smaller $\beta$, as large a dynamic aperture with a much stronger beam-beam effect, and a very small momentum compaction factor. Tungsten shielding and/or open midplane bending magnets are needed to keep the large heat load from decay electrons from quenching superconducting magnets.

\section{Critical Issues}

Normal-conducting RF cavities operating in high magnetic fields are needed in the Muon Collider for capture, bunching, phase rotation, and cooling. The maximum stable gradient degrades quickly with magnetic field. Multiple studies are being carried out at the Fermilab MuCool Test Area to find ways to increase the maximum magnetic field: reducing/eliminating field emission, materials studies, RF cavities filled with high-pressure gas $\left(\mathrm{H}_{2}\right)$, and magnetic insulation. 
Other critical issues include incorporating neutrino radiation mitigation into the ring design, R\&D on very-high-field and fast-ramping magnets, end-to-end simulation of the complete Muon Collider, space charge and wake field questions, and successful completion of the MICE experiment.

\section{MuCool Test Area}

The MuCool Test Area (MTA) at Fermilab is a dedicated facility to support component development. The facility provides an experimental hall, RF power, a cryogenics plant, and a high-intensity $400-\mathrm{MeV}$ proton beam from the linac. Absorbers, magnets, and 201- and 805MHz RF cavities are tested. The MTA supports the MICE experiment. Crucial tests of the limits on the accelerating gradient in normal-conducting RF cavities in high magnetic fields are being carried out at the MTA.

\section{Summary and Conclusions}

There has been considerable progress on Muon Collider R\&D, but many challenges remain. The Muon Accelerator Program (MAP) was approved by the U.S. Department of Energy. Within 5-6 years we will have a Design Feasibility Study and cost range for a muti-TeV Muon Collider. The IDS-NF Reference Design Report will be completed. The decision on the energy for the next lepton collider will depend on results from the LHC.

\section{Acknowledgements}

The author wishes to acknowledge the collaboration with many colleagues in MAP and support from the U.S. Department of Energy under grant number DE-FG02-07ER41487.

\section{References}

[1] Presentations at Muon Collider 2011 Physics-Detectors-Accelerators, June 27 - July 1, 2011, Telluride, Colorado, http://conferences.fnal.gov/muon11/.

[2] See http://map.fnal.gov/.

[3] S. Choubey et al., IDS-NF Collaboration Interim Design Report, Technical Note IDS-NF-020 (2011), https://www.ids-

nf.org/wiki/FrontPage/Documentation?action=AttachFile\&do=get\&target $=$ IDS-NF-020-v1.0.pdf.

[4] G. Gregoire et al., An International Muon Ionization Cooling Experiment (MICE), Proposal to the Rutherford Appleton Laboratory, MICE-NOTE-GEN-0021 (2003).

[5] K. McDonald et al., The MERIT high-power target experiment at the CERN PS, in proceedings of IPAC 2010, Kyoto, Japan, p. 3527.

[6] R\&D Proposal for the National Muon Accelerator Program, FERMILAB-TM-2459-APC, and references therein (2010),

http://map.fnal.gov/proposals/pdfs/MAPproposal-R6d.pdf. 